\title{
Comparative antimicrobial efficacy of selected root canal irrigants on commonly isolated microorganisms in endodontic infection
}

\author{
Sandeep Dubey ${ }^{1}$, Suparna Ganguly Saha ${ }^{1}$, Balakrishnan Rajkumar ${ }^{2}$, Tapan Kumar Dhole ${ }^{3}$
}

Correspondence: Dr. Sandeep Dubey

Email: sandeepdubey.mds@gmail.com

\author{
'Department of Conservative Dentistry and \\ Endodontics, College of Dental Sciences and Hospital, \\ Indore, Madhya Pradesh, India, \\ 2Department of Conservative Dentistry and \\ Endodontics, Babu Banarasi Das College of Dental \\ Sciences, Lucknow, Uttar Pradesh, India, \\ ${ }^{3}$ Department of Microbiology, Sanjay Gandhi \\ Postgraduate Institute of Medical Sciences, Lucknow, \\ Uttar Pradesh, India
}

\section{ABSTRACT}

Objective: This study aims to evaluate and compare the antimicrobial efficacy of three selected root canal irrigants (BioPure MTAD, metronidazole, aztreonam) against microbes commonly isolated from polymicrobial microbiota of root canal infection. Materials and Methods: This study was designed with four experimental groups (Group I - Bacteroides fragilis, Group II - Propionibacterium acnes, Group III - Enterococcus faecalis, Group IV - Candida albicans) based on the microbes selected for the study. Group I and Group II bacteria were used to compare and evaluate antimicrobial effect of BioPure MTAD, metronidazole, aztreonam, and normal saline. Group III and Group IV bacteria were used to compare and evaluate antimicrobial efficacy of BioPure MTAD, aztreonam, and normal saline. Normal saline was used as a control irrigant in this study. Agar disc diffusion method was applied to assess and compare the antimicrobial action of selected irrigants. Results: Metronidazole was found to be the most effective root canal irrigant against $B$. fragilis and P. acnes among the tested irrigants. Mean zone of inhibition against $E$. faecalis has been shown to be maximum by BioPure MTAD, followed by aztreonam. Antifungal effect against C. albicans was only shown by BioPure MTAD. Conclusions: Overall, BioPure MTAD is the most effective root canal irrigant as it has shown an antibacterial effect against all the tested microorganisms. However, metronidazole showed maximum antibacterial effect against obligate anaerobes. Aztreonam also showed an antibacterial effect in the present study, raising its possibility to be used as a root canal irrigant in the future.

Key words: Agar disc diffusion method, BioPure MTAD, Candida albicans, Enterococcus faecalis

\section{INTRODUCTION}

Reduction or elimination of microflora during root canal treatment is achieved by the combined effect of both chemomechanical preparation and intracanal medications. ${ }^{[1,2]}$ The removal of microorganisms and other irritants from the confines of the root canal

\begin{tabular}{|l|l|}
\hline \multicolumn{2}{|c|}{ Access this article online } \\
\hline Quick Response Code: \\
\hline
\end{tabular}

is conducted by means of the mechanical action of instruments and the flow and backflow of the irrigating solutions. ${ }^{[3,4]}$

This is an open access article distributed under the terms of the Creative Commons Attribution-NonCommercial-ShareAlike 3.0 License, which allows others to remix, tweak, and build upon the work non-commercially, as long as the author is credited and the new creations are licensed under the identical terms.

For reprints contact: reprints@medknow.com

How to cite this article: Dubey S, Saha SG, Rajkumar B, Dhole TK.
Comparative antimicrobial efficacy of selected root canal irrigants on
commonly isolated microorganisms in endodontic infection. Eur J Dent
$2017 ; 11: 12-6$.
DOI: $10.4103 /$ ejd.ejd_141_16
commonly isolated microorganisms in endodontic infection. Eur J Dent DOI: 10.4103/ejd.ejd_141_16 
BioPure MTAD (Dentsply/Tulsa, Tulsa, OK, USA), which is a mixture of a tetracycline isomer, citric acid, and a detergent, ${ }^{[5]}$ has been recommended as a final rinse irrigant because of its antimicrobial properties and its ability to remove the smear layer. ${ }^{[6,7]}$ It is less cytotoxic than most of the endodontic medicaments. ${ }^{[8,9]}$

Aztreonam, which is a synthetic monocyclic beta-lactam antibiotic, has a molecular structure different from older beta-lactam antibiotics as it has a less complex nucleus. ${ }^{[10]}$ It has shown excellent activity against Gram-negative bacilli. ${ }^{[11]}$

Metronidazole, a nitroimidazole compound, is a broad-spectrum antibiotic and exhibits activity against anaerobic bacteria and protozoa. It demonstrates effective antibacterial activity against anaerobic cocci as well as Gram-negative and Gram-positive bacilli. In the treatment of periodontal disease, it has been used both topically and systemically. ${ }^{[12]}$

The objective of this research was to assess and compare the antibacterial and antifungal effectiveness of BioPure MTAD, aztreonam, and metronidazole against obligate anaerobic bacteria, i.e., Bacteroides fragilis and Propionibacterium acnes, facultative anaerobic bacteria, i.e., Enterococcus faecalis, and yeast, i.e., Candida albicans.

\section{MATERIALS AND METHODS}

The microorganisms selected for this study, i.e., B. fragilis ATCC 25285 (Group 1), P. acnes ATCC 6921 (Group 2), E. faecalis ATCC 29212 (Group 3), and C. albicans ATCC 10231 (Group 4), were obtained from the American type culture collection and stored at a temperature of $-10^{\circ} \mathrm{C}$ to $-20^{\circ} \mathrm{C}$ in a freezer.

Strains of the selected microorganisms were reactivated in the respective media at a given temperature and time.

Groups 1 and 2 - Activated in brain-heart infusion broth supplemented with hemin and menadione at $37^{\circ} \mathrm{C}$ for $48 \mathrm{~h}$.

Group 3 - Activated in brain-heart infusion broth for $24 \mathrm{~h}$ at $37^{\circ} \mathrm{C}$.

Group 4 - Activated in Sabouraud dextrose agar broth at $37^{\circ} \mathrm{C}$ for $24 \mathrm{~h}$.

After the revival of microorganisms, four to five well-isolated colonies of each strain were picked up with a sterile loop, later dissolving them in respective test tubes containing $0.85 \%$ of $5 \mathrm{ml}$ sterile saline to create a turbidity of $0.5 \mathrm{McF}$ arland scale $\left(1.5 \times 10^{8} \mathrm{CFU} / \mathrm{ml}\right)$ for the preparation of inoculum. The resulting suspension was then spread on agar plates with the help of a sterile cotton swab in a form so that a lawn growth was observed.

The media used for the four groups were as follows:

Group 1 - Wilkins-Chalgren agar plate (HiMedia, Mumbai).

Group 2 - Brain-heart infusion medium (HiMedia, Mumbai) with $1 \%$ glucose.

Group 3 - Mueller-Hinton agar plate (HiMedia, Mumbai).

Group 4 - Sabouraud dextrose agar plate (HiMedia, Mumbai).

\section{Antibiotic disc preparation}

Antibiotic solution of BioPure MTAD was prepared by mixing powder and diluent.

Sterilized Whatman paper no. 1 was used to prepare 6-mm diameter discs, which were later soaked in $40 \mu \mathrm{l}$ of normal saline and BioPure MTAD, respectively.

The company-made aztreonam and metronidazole antibiotic discs (BD Diagnostic, India) were also used for the test.

These antibiotic discs were then aseptically transplanted to the agar plate formerly incubated with microorganisms.

For Group 1 and Group 2, agar plates BioPure MTAD, aztreonam, metronidazole, and normal saline containing discs, and for Group 3 and Group 4, agar plates, BioPure MTAD, aztreonam, and normal saline prepared discs were transferred aseptically.

Group 1 and Group 2 agar plates were incubated in an anaerobic environment created using the Anoxomat system (Mart Microbiology BV, Netherlands) for $48 \mathrm{~h}$.

Group 3 and Group 4 plates were incubated for $48 \mathrm{~h}$ at $37^{\circ} \mathrm{C}$ under the aerobic conditions, in an atmosphere of $10 \% \mathrm{CO}_{2}$, in a $\mathrm{CO}_{2}$ incubator (New Brunswick, USA). All assays were repeated five times. Measurement of zones of inhibition was conducted by a Vernier caliper. 


\section{RESULTS}

The results obtained were statistically evaluated using one-way ANOVA test and the intergroup comparison was done using Bonferroni multiple comparison test. Value of $P<0.001$ was considered statistically significant.

- Bacteroides fragilis (Group 1) [Tables 1a and b]

- Propionibacterium acnes (Group 2) [Tables 2a and b]

- Enterococcus faecalis (Group 3) [Tables 3a and b]

- Candida albicans (Group 4) [Tables 4a and b].

\section{DISCUSSION}

Disc diffusion technique, a well-established method for antimicrobial research, has been applied in this study. ${ }^{[13,14]}$ This method has an advantage that chemical properties of tested medicaments do not alter during the procedure and also it is less technique sensitive. ${ }^{[15]}$

Anoxomat system was used for anaerobic culture in the current study as earlier research ${ }^{[16]}$ had shown that with the use of this system commendable growth is achieved, in terms of density and colony size. Evidently, the Anoxomat method is considerably reliable and appears to support the growth of strict anaerobes efficiently. Zone of inhibition around specimens was measured in the present study using a Vernier caliper at three different points as done in previous studies..$^{[17,18]}$ In the present study, all assays were repeated five times to establish reproducibility. ${ }^{[19]}$

Tabulated results demonstrated that metronidazole produced the maximum zone of inhibition against B. fragilis [Graph 1]. Vijaykumar et al. ${ }^{[20]}$ and Whelan et al. ${ }^{[21]}$ demonstrated that metronidazole was effective against $B$. fragilis, which is in agreement with the results obtained in the current study.

Antimicrobial efficacy against $P$. acnes in the existing study was shown to be maximum by metronidazole [Graph 2]. Effect of metronidazole on P. acnes has been reported with mixed findings in the literature. Gaetti-Jardim Júnior et al. ${ }^{[22]}$ determined the positive antibacterial effect of metronidazole against $P$. acnes, and the results obtained were in accordance with the present study. Similarly, Effat Khodeinae et al. ${ }^{[23]}$ demonstrated that $2 \%$ metronidazole showed adequate efficacy against $P$. acnes while evaluating the efficacy of $2 \%$ metronidazole gel against acne vulgaris. In contrast to the above results, few studies reveal no effect of metronidazole against $P$. acnes. ${ }^{[24]}$

\begin{tabular}{|c|c|c|}
\hline Irrigants & Zone of inhibition $(\mathrm{mm})$, mean $\pm S D$ & Effect \\
\hline BioPure MTAD & $26.75 \pm 1.02$ & Sensitive \\
\hline Metronidazole & $30.00 \pm 1.63$ & Sensitive \\
\hline Aztreonam & $28.25 \pm 1.02$ & Sensitive \\
\hline Normal saline & $0 \pm 0.0$ & Resistant \\
\hline
\end{tabular}

Table 1b: Intergroup comparison of mean zone of inhibition size for the irrigants against Bacteroides fragilis

\begin{tabular}{lccl}
\hline & $\boldsymbol{t}$ & $\boldsymbol{P}$ & Significance \\
\hline 1 versus 2 & 4.22 & $<0.001$ & Significant \\
1 versus 3 & 1.94 & 0.0 & Nonsignificant \\
1 versus 4 & 34.71 & $<0.001$ & Significant \\
2 versus 3 & 2.27 & 0.0 & Nonsignificant \\
2 versus 4 & 38.93 & $<0.001$ & Significant \\
3 versus 4 & 36.66 & $<0.001$ & Significant \\
\hline 1 - BioPure MTAD, 2 - Metronidazole, 3 - Aztreonam, 4 - Normal saline
\end{tabular}

\begin{tabular}{|c|c|c|}
\hline Irrigants & Zone of inhibition $(\mathrm{mm})$, mean \pm SD & Effect \\
\hline BioPure MTAD & $26.00 \pm 0.82$ & Sensitive \\
\hline Metronidazole & $29.25 \pm 1.82$ & Sensitive \\
\hline Aztreonam & $0 \pm 0.0$ & Resistant \\
\hline Normal saline & $0 \pm 0.0$ & Resistant \\
\hline
\end{tabular}

Table 2b: Intergroup comparison of mean zone of inhibition size of irrigants against Propionibacterium acnes

\begin{tabular}{lccl}
\hline & $\boldsymbol{t}$ & $\boldsymbol{P}$ & Significance \\
\hline 1 versus 2 & 7.96 & $<0.001$ & Significant \\
1 versus 3 & 63.68 & $<0.001$ & Significant \\
1 versus 4 & 63.68 & $<0.001$ & Significant \\
2 versus 3 & 71.64 & $<0.001$ & Significant \\
2 versus 4 & 71.64 & $<0.001$ & Significant \\
3 versus 4 & 0.0 & 0.0 & Nonsignificant \\
\hline
\end{tabular}

1 - BioPure MTAD, 2 - Metronidazole, 3 - Aztreonam, 4 - Normal saline

Table 3a: Effect of root canal irrigants and their values
of zone of inhibition against Enterococcus Faecalis
\begin{tabular}{lcc}
\hline Irrigants & Zone of inhibition (mm), mean \pm SD & Effect \\
\hline BioPure MTAD & $29.25 \pm 1.63$ & Sensitive \\
Aztreonam & $8.5 \pm 1.23$ & Sensitive \\
Normal saline & $0.0 \pm 0.0$ & Resistant \\
\hline ANOVA F=516.12, $P<0.001$ (significant). SD: Standard deviation &
\end{tabular}

Metronidazole is a potent bactericidal, which is efficacious against anaerobes that are composed of 


\begin{tabular}{|c|c|c|c|}
\hline & $t$ & $P$ & Significance \\
\hline 1 versus 2 & 24.9 & $<0.001$ & Significant \\
\hline 1 versus 3 & 35.10 & $<0.001$ & Significant \\
\hline 2 versus 3 & 10.20 & $<0.001$ & Significant \\
\hline
\end{tabular}

Table 4a: Effect of root canal irrigants and their values of zone of inhibition against Candida albicans

\begin{tabular}{lcl}
\hline Irrigants & Zone of inhibition $(\mathrm{mm})$, mean $\pm S D$ & Effect \\
\hline BioPure MTAD & $8.50 \pm 0.82$ & Sensitive \\
Aztreonam & $0.0 \pm 0.0$ & Resistant \\
Normal saline & $0.0 \pm 0.0$ & Resistant \\
\hline ANOVA F $=430.50, P<0.001$ (significant). SD: Standard deviation &
\end{tabular}

Table 4b: Intergroup comparison of mean zone of inhibition size of irrigants against Candida albicans

\begin{tabular}{lccl}
\hline & $\boldsymbol{t}$ & $\boldsymbol{P}$ & Significance \\
\hline 1 versus 2 & 25.50 & $<0.001$ & Significant \\
1 versus 3 & 25.50 & $<0.001$ & Significant \\
2 versus 3 & 00.00 & 0.00 & Nonsignificant \\
\hline 1 - BioPure MTAD, 2 - Aztreonam, 3 - Normal saline &
\end{tabular}

electron transport components, i.e., ferredoxin, which are capable of donating electrons to metronidazole, developing highly reactive nitro radical anions that kill susceptible organisms by a radical-mediated mechanism. ${ }^{[18]}$ This could be a possible reason for its high antimicrobial efficacy against $B$. fragilis and $P$. acnes.

In the present study, metronidazole was not used to test antimicrobial efficacy for E. faecalis and C. albicans because it is ineffective against facultative anaerobes. Literature has already proven its lacks of antimicrobial effect against E. faecalis. ${ }^{[19]}$ Krishna et al. ${ }^{[25]}$ in their study demonstrated that $10 \%$ metronidazole was not effective against E. faecalis among the tested intracanal medicaments. Yujra et al. ${ }^{[26]}$ demonstrated that long-standing metronidazole therapy preferred the establishment of $C$. albicans in the oral cavity of rats.

In the current study, maximum mean zone of inhibition against $E$. faecalis was revealed by BioPure MTAD [Graph 3], which is in accordance with several other studies. ${ }^{[7]}$ Newberry et al..$^{[8]}$ in their study identified that seven out of the eight tested strains of E. faecalis growth were affected when MTAD was used as a final irrigant. The presence of doxycycline defines the superior antimicrobial effect of MTAD against E. faecalis..$^{[9]}$ However, results of this research are in

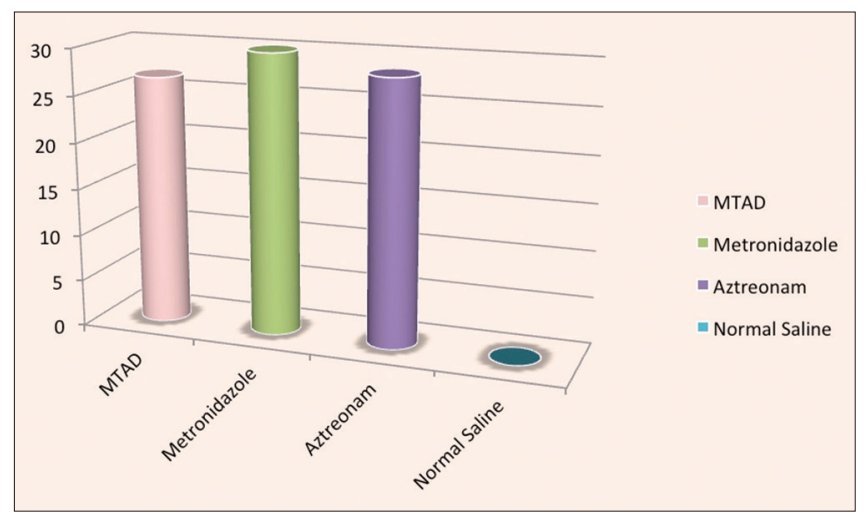

Graph 1: Comparison of mean zone of inhibition of root canal irrigants against Bacteroides fragilis

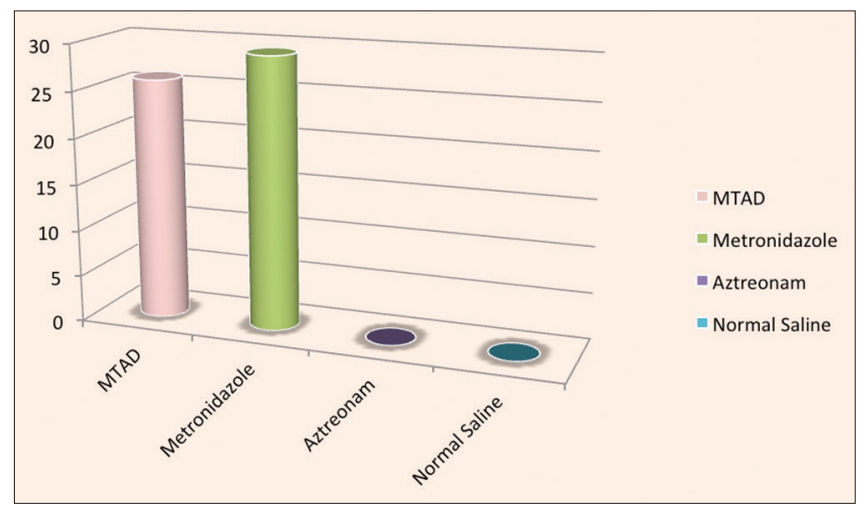

Graph 2: Comparison of mean zone of inhibition of root canal irrigants against Propionibacterium acnes

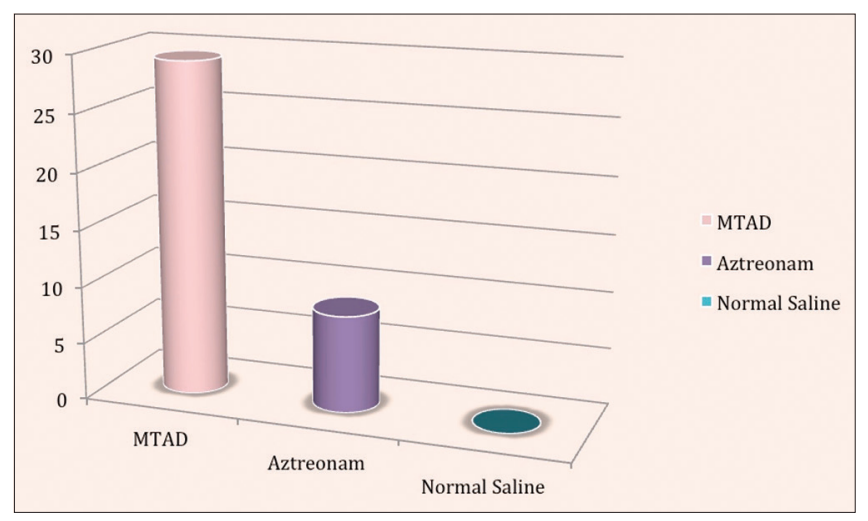

Graph 3: Comparison of mean zone of inhibition of root canal irrigants against Enterococcus faecalis

contradiction to reports which refute the antimicrobial effect of MTAD against E. faecalis. ${ }^{[10]}$

In the present study, antifungal effect against C. albicans was shown only by MTAD this result obtained was in agreement with the result given by Arslan et al., ${ }^{[27]}$ who demonstrated antimicrobial action of MTAD against $C$. albicans and $E$. faecalis. The antifungal effect of MTAD can be attributed to the better flow and penetration properties of doxycycline. ${ }^{[12]}$ Contrary to 
the above results, a study conducted by Ashari et al..$^{[28]}$ showed that MTAD is ineffective against $C$. albicans and its substantivity may be altered when used in conjunction with $\mathrm{NaOCl}$.

MTAD in the current research has revealed substantial zone of inhibition against both $B$. fragilis and P. acnes. This may be possibly due to the occurrence of doxycycline as earlier research has confirmed the action of doxycycline on $B$. fragilis and $P$. acnes ${ }^{[11]}$ in inhibiting the protein synthesis.

Aztreonam in the present study showed significant antimicrobial efficacy against $B$. fragilis and mild antibacterial activity against $E$. faecalis but was completely inactive against $P$. acnes and C. albicans microorganisms. Aztreonam, a bactericidal antibiotic, acts by interrupting with the formation of the bacterial cell wall. ${ }^{[2]}$ It causes lysis and death of Gram-negative bacteria by binding to the penicillin-binding protein-3 (PBP-3), ${ }^{[3]}$ which might be the reason for its activity against $B$. fragilis. Poor affinity has been shown for the PBP-3 of Gram-positive and anaerobic bacteria which could be the reason behind its mild activity against $E$. faecalis.

\section{CONCLUSIONS}

On the basis of results obtained in the current study, it may be stated that BioPure MTAD can be recommended as an effective alternative to the currently used root canal irrigants due to its superior antimicrobial properties against both aerobic and anaerobic microbial organisms as well as C. albicans. Metronidazole and aztreonam, on the other hand, are, however, not significantly efficacious against all strains of microbes commonly present in the root canal, thus limiting their use as an effective antimicrobial root canal irrigant.

Although the present study was done with strict adherence to all the scientific protocols, still further studies are required to evaluate the efficacy of the different irrigants, which may further widen their horizon in the field of endodontics.

\section{Financial support and sponsorship \\ Nil.}

\section{Conflicts of interest}

There are no conflicts of interest.

\section{REFERENCES}

1. Kargül B, Tanboga I, Altinok B. Conventional endodontic treatment of primary molars using metronidazole as an intra-canal medicament: A pilot study. Eur Arch Paediatr Dent 2010;11:196-200.
2. Jacobus NV, Ferreira MC, Barza M. In vitro activity of azthreonam, a monobactam antibiotic. Antimicrob Agents Chemother 1982;22:832-8.

3. Antonio-Velmonte MM, Santana RT, Manalastas RM. Efficacy and safety of Aztreonam in the treatment of nosocomial gram negative bacterial infections. Phil J Microbiol Infect Dis 1989;18:53-7.

4. Demiryurek EO, Onuk EE, Yuksel G, Ciftci A. Evaluation of microbial contamination of resilon and gutta-percha cones and their antimicrobial activities. Afr J Microbiol Res 2012;6:6275-80.

5. Torabinejad M, KhademiAA, Babagoli J, Cho Y, Johnson WB, Bozhilov K. A new solution for the removal of the smear layer. J Endod 2003;29:170-5.

6. Ando N, Hoshino E. Predominant obligate anaerobes invading the deep layers of root canal dentin. Int Endod J 1990;23:20-7.

7. Sanjiwan R, Chandra S, Jaiswal JN, Mastan. The effect of metronidazole on the anaerobic microorganisms of the root canal - A clinical study. Fed Oper Dent 1990;1:30-6.

8. Newberry BM, Shabahang S, Johnson N, Aprecio RM, Torabinejad $\mathrm{M}$. The antimicrobial effect of biopure MTAD on eight strains of Enterococcus faecalis. J Endod 2007;33:1352-4.

9. Brauner AW, Conrads G. Studies into the microbial spectrum of apical periodontitis. Int Endod J 1995;28:244-8.

10. Waltimo TM, Sirén EK, Torkko HL, Olsen I, Haapasalo MP. Fungi in therapy-resistant apical periodontitis. Int Endod J 1997;30:96-101.

11. Le Goff A, Bunetel L, Mouton C, Bonnaure-Mallet M. Evaluation of root canal bacteria and their antimicrobial susceptibility in teeth with necrotic pulp. Oral Microbiol Immunol 1997;12:318-22.

12. Abou-Rass M, Bogen G. Microorganisms in closed periapical lesions. Int Endod J 1998;31:39-47.

13. Tronstad L, Sunde PT. The evolving new understanding of endodontic infections. Endod Topics 2003;6:57-77.

14. Gajan EB, Aghazadeh M, Abashov R, Salem Milani A, Moosavi Z. Microbial flora of root canals of Pulpally-infected teeth: Enterococcus faecalis a prevalent species. J Dent Res Dent Clin Dent Prospects 2009;3:24-7.

15. Chavez De Paz L. Gram-positive organisms in endodontic infections. Endod Topics 2004;9:79-96.

16. Siqueira JF Jr., Sen BH. Fungi in endodontic infections. Oral Surg Oral Med Oral Pathol Oral Radiol Endod 2004;97:632-41.

17. Sood S, Malhotra M, Das BK, Kapil A. Enterococcal infections \& antimicrobial resistance. Indian J Med Res 2008;128:111-21.

18. Ashraf H, Samiee M, Eslami G, Ghodse Hosseini MR. Presence of Candida albicans in root canal system of teeth requiring endodontic retreatment with and without periapical lesions. Iran Endod J 2007;2:24-8.

19. Waltimo TM, Sen BH, Meurman JH, Ørstavik D, Haapasalo MP. Yeasts in apical periodontitis. Crit Rev Oral Biol Med 2003;14:128-37.

20. Vijaykumar S, Gunashekhar M, Himagiri S. In vitro effectiveness of different endodontic irrigants on the reduction of Enterococcus faecalis in root canals. J Clin Exp Dent 2010;2:e169-72.

21. Whelan JPF, Hale JH. Bactericidal activity of metronidazole against Bacteroides fragilis. J Clin Pathol 1973;26:393-95.

22. Gaetti-Jardim Junior E, Landucci LF, Lins SA, Vieira EM, de Oliveira SR. Susceptibility of strict and facultative anaerobes isolated from endodontic infections to metronidazole and betalactams. J Appl Oral Sci 2007;15:539-45

23. Khodaeiani E, Fouladi RF, Yousefi N, Amirnia M, Babaeinejad S, Shokri J. Efficacy of $2 \%$ Metronidazole gel in moderate Acne Vulgaris. Indian J Dermatol 2012;57:279-81.

24. Kamberi B, Bajrami D, Stavileci M, Omeragiq S, Dragidella F, Koçani F. The antibacterial efficacy of biopure MTAD in root canal contaminated with Enterococcus faecalis. ISRN Dent 2012;2012:390526.

25. Krishna JN, Raghu R, Bolla N, Muddanna K. An in vitro comparative evaluation of the antimicrobial efficacy of $10 \%$ metronidazole gel, $2 \%$ chlorhexidine gel, and a combination of calcium hydroxide and $2 \%$ chlorhexidine gel against Enterococcus faecalis. J Orofac Sci 2012;4:26-31

26. Yuira VQ, Scherma AP, Junqueira JC. The role of metronidazole on the establishment and persistence of oral candidosis. Braz J Oral Sci 2006;5:1041-47.

27. Arslan S, Ozbilge H, Kaya EG, Er O. In vitro antimicrobial activity of propolis, biopure MTAD, sodium hypochlorite and chlorhexidine on Enterococcus faecalis and Candida albicans. Saudi Med J 2011;32:479-83.

28. Asna Ashari M, Fayaz F, Moezzi Ghadim N, Alim Marvasti L, Mehrabi Y. Evaluation of the antimicrobial effects of MTAD, NaOCl against selected endodontic pathogens. Iran Endod J 2009;4:63-8. 